\title{
The Role and Mechanism of MALATI Long Non-Coding RNA in the Diagnosis and Treatment of Head and Neck Squamous Cell Carcinoma
}

\author{
Dong Ye (D) \\ Yongqin Deng ${ }^{2}$ \\ Zhisen Shen (1D) \\ 'Department of Otorhinolaryngology- \\ Head and Neck Surgery, Lihuili Hospital \\ of Ningbo University, Ningbo, 315040, \\ Zhejiang, People's Republic of China; \\ ${ }^{2}$ Department of Otorhinolaryngology- \\ Head and Neck Surgery, Ningbo \\ University School of Medicine, Ningbo, \\ 3I52 II, People's Republic of China
}

Correspondence: Dong Ye; Zhisen Shen Department of OtorhinolaryngologyHead and Neck Surgery, Lihuili Hospita of Ningbo University, Ningbo, 315040, People's Republic of China Tel +86-1381986/213; +86-13906687216 Fax +86-574-87392232

Email yedong518@sina.com; szs7216@sina.com

\begin{abstract}
Head and neck squamous cell carcinoma (HNSCC) is the most common malignant tumor of the head and neck. HNSCC mainly affects the oral cavity and the laryngeal, laryngopharyngeal, and oropharyngeal tracts. The high incidence, hidden onset, low survival rate, and unsatisfactory effects of treatment effect underscore the importance of identify the mechanisms of HNSCC occurrence and development. Although there is a very urgent need for early diagnosis and treatment, there are currently no reliable early HNSCC diagnosis biomarkers or effective treatment targets. Long non-coding RNA (lncRNA) is widely involved in biological processes, especially as a key regulator of tumorigenesis and development. Lung adenocarcinoma metastasis-associated transcript 1 (MALAT1) is an important member of the lncRNA family that can regulate the occurrence and development of a variety of malignant tumors and is anticipated to be an ideal marker for early tumor diagnosis and an effective therapeutic target. Here, we review the research progress into the role of MALAT1 in the diagnosis and treatment of HNSCC and its regulatory mechanism.
\end{abstract}

Keywords: head and neck squamous cell carcinoma, MALAT1, diagnosis, treatment, mechanism

\section{Introduction}

Head and neck squamous cell carcinoma (HNSCC) is the most common malignant tumors of the head and neck, and its incidence is 6th among all cancers with 890,000 new cases and 450,000 deaths in 2018. The incidence of HNSCC continues to rise and is anticipated to increase by $30 \%$ (ie, 1.08 million new cases annually) by $2030 .{ }^{1}$ HNSCCs mainly including laryngeal cancer, hypopharyngeal cancer, oral cancer, and oropharyngeal cancer. $^{2-4}$ Most patients have already developed local progression at the time of diagnosis. The tumors are characterized by high mortality and tolerance to radiotherapy and chemotherapy, and the 5-year overall survival rate of patients with HNSCC is only 50\%. ${ }^{5,6}$ Although there are many theories about the etiology of HNSCC, the mechanism of its occurrence and development remains unclear. ${ }^{7}$ Surgical treatment or comprehensive treatment based on radiotherapy and chemotherapy are the main treatments, but the curative effect is not satisfactory. ${ }^{8}$ Therefore, exploring HNSCC-related biomarkers as reliable early diagnosis markers, prognostic indicators, and treatment targets, and investigating possible mechanisms of action are of great significance for the early HNSCC detection. Such information will guide individualized treatment and improve patient survival. 
Long noncoding RNA (lncRNA) plays an important regulatory role in the biology of head and neck tumors. ${ }^{9}$ IncRNAs can act tumor-promoting genes to promote the growth, invasion, and migration of cancer cells, or they can serve as tumor suppressor genes to inhibit head and neck tumor occurrence and development. ${ }^{10,11}$ Various lncRNAs have different mechanisms and regulatory targets in the occurrence, development, and metastasis of head and neck tumors. IncRNAs can regulate gene transcription by binding to enzymes or protein complexes, can participate in chromosome remodeling and epigenetic regulation, and can also inhibit microRNA (miRNA) or alter mRNA modifications to regulate mRNA transcription and translation. ${ }^{12-14}$ Unique cross-regulation between lncRNA and miRNA was recently described, and emerging evidence shows that such crosstalk has a marked effect on human cancer metastasis, partially through epithelialmesenchymal transition (EMT) regulation. ${ }^{15}$ The IncRNAs already found to regulate head and neck tumors can be divided into three major mechanistic categories: cancer-promoting, cancer-suppressing, and dual mechanism of action. Li et al ${ }^{16}$ discussed the role and function of lncRNAs in laryngeal squamous cell carcinoma (LSCC) proliferation, invasion, and apoptosis and analyzed the relationship between lncRNAs and lncRNA-regulated signaling pathways in LSCC pathogenesis. It provides reference ideas for molecular mechanism research into LSCC that targets lncRNA and its signaling pathways and will contribute to the development of clinical prevention measures therapeutic drugs, and individualized treatment, thereby improving patient quality of life.

Akbari Dilmaghani et $\mathrm{al}^{17}$ reviewed the vital regulatory roles of lncRNAs and introduced their roles in progression of head and neck cancer subtypes.

Meng et $\mathrm{al}^{18}$ described molecular mechanisms of upor down-regulation of IncRNAs and expounded their functions in HNSCC pathology and clinical practices. They also highlighted their potential clinical applications as biomarkers for HNSCC diagnosis, prognosis, and treatment.

Several studies have shown that treatments based on non-coding RNAs (ncRNAs) - including lncRNAs - may hold promise as alternative methods for treating oral premalignant disorders and oral squamous cell carcinoma (OSCC). Their use as therapeutic agents appear promising. ${ }^{19}$

Metastasis-associated lung adenocarcinoma transcript1 (MALAT1) is an important member of the lncRNA family located on chromosome 11q13.1. MALAT1 was first discovered in non-small cell lung carcinoma (NSCLC) and is involved in the regulation of cell cycle and migration. ${ }^{20,21}$ MALAT1 is unregulated in head and neck malignant tumors; lung, liver, colon, breast, and gastric cancers; and other malignant tumors. MALAT1 expression is closely related to tumor size, stage, and poor prognosis and can be used to evaluate clinicopathological characteristics and prognosis in tumor patients. ${ }^{22-24}$ Here, we review the role of MALAT1 in the diagnosis and treatment of HNSCC and discuss its possible mechanism.

\section{MALATI Structure and Function}

MALAT1 is located at 11q13.1 and has 8700 nucleotides. It is an lncRNA that is expressed in the nucleus in high abundance. MALAT1 is highly conserved in mammals, is widely expressed in normal human tissues and cells, and has obvious patterns of tissue specific and temporal expression. $^{25,26}$ MALAT1 was originally identified in human NSCLC and is abundant in lung cancer tissues. MALAT1 expression is positively correlated with lung cancer metastasis and negatively correlated with prognosis; it is an important prognostic parameter for patients with stage I lung adenocarcinoma or squamous cell carcinoma. ${ }^{27}$ MALAT1 plays a critical role in transcription and cell cycle regulation, epigenetics, inflammation, and tumor metastasis. ${ }^{28,29}$ MALAT1 expression is significantly higher in a variety of tumor tissues compared to normal tissues and is associated with tumor metastasis. MALAT1 has a regulatory effect on the occurrence and development of several tumors, including laryngeal, hypopharyngeal, thyroid, esophageal, lung, liver, and ovarian cancers. ${ }^{30-36}$

\section{The Relationship Between MALAT I and HNSCC}

MALAT1 can regulate the occurrence and development of a variety of malignant tumors. It also plays roles in transcription, cell cycle regulation, epigenetics, and tumor metastasis. In recent years, there has been an increasing amount of research into the growth promotion effect of MALAT1 on HNSCC, highlighting the importance of MALAT1 for tumor diagnosis, prognosis evaluation, and targeted therapy. MALAT1 may function as an oncogene to regulate HNSCC growth, apoptosis, invasion, and metastasis. A number of studies $^{32,37,38}$ confirmed that MALAT1 expression was significantly up-regulated in HNSCC tissues including laryngeal, 
nasopharyngeal, and oral samples. MALAT1 is expected to be a marker for early tumor diagnosis and prognosis. Low MALAT1 expression can inhibit the proliferation and metastasis of a variety of HNSCC cells, play a role in suppressing cancer, and improve sensitivity to chemotherapeutics. Therefore, MALAT1 is expected to serve as a new target for tumor treatment.

\section{The Role of MALATI in HNSCC Diagnosis and Progression/Prognosis}

In-depth study of the correlation between MALAT1 and HNSCC as a diagnostic tool has revealed the characteristics of high accuracy, efficiency, and minimal invasiveness. Although MALAT1 expression has potential value in HNSCC diagnosis, this application is still in the research stage. However, preliminary studies suggest that MALAT1 can be used as an ideal marker for early HNSCC diagnosis.

Yao et $\mathrm{al}^{22}$ showed that MALAT1, HOXA11-AS, and LINC00964 lncRNAs are expected to become potential circulating biomarkers for early HNSCC diagnosis. Among these, MALAT1 is significantly dysregulated in laryngeal squamous cell carcinoma (LSCC) specimens, indicating that it is involved in LSCC occurrence and development, ${ }^{32}$ but further studies are required to evaluate the clinical role of MALAT1 as a potential diagnostic and prognostic biomarker of LSCC. He et $\mathrm{al}^{37}$ showed that circulating MALAT1, AFAP1-AS1, and AL359062 may be useful as new serum biomarkers for the diagnosis and evaluation of nasopharyngeal carcinoma.

MALAT1 is upregulated in tongue squamous cell carcinoma (TSCC) tissues and is associated with differentiation and clinical stage, suggesting that it can be used as an independent biomarker to assess TSCC prognosis. ${ }^{39}$ Understanding the critical role of MALAT1 in TSCC may lead to the development of a novel diagnostic marker for this type of cancer. ${ }^{39}$ Elevated MALAT1 expression in OSCC may play a role in tumorigenesis and metastasis, but further studies are needed to determine which mechanisms affect the growth and migration of MALAT1 in human TSCC SCC4 cells and verify that MALAT1 expression is increased in patients with OSCC. ${ }^{38}$

The above studies demonstrated that MALAT1 expression levels in patients with HNSCC are correlated with tumor occurrence, development, and prognosis. For most types of HNSCC, higher MALAT1 expression is associated with worse prognosis. However, individual research results indicate that MALAT1 can have biological effects contrary to those mentioned above. Studies by Vishwakarma and others showed that MALAT1 expression is significantly down-regulated in OSCC tissues compared with neighboring normal tissues. ${ }^{40}$

Cisplatin (DDP) is the first-line chemotherapy agent for OSCC. DDP resistance leads to lower drug efficacy and survival benefits. DDP-resistant cells showed higher MALAT1 expression compared to DDP-naïve cells. MALAT1 overexpression in DDP-naïve cells enhanced drug resistance and suppressed apoptosis in OSCC cells. MALAT1 knockdown in DDP-resistant cells induced apoptotic cell death and restored the sensitivity to DDP. Further analyses suggested that MALAT1 might promote DDP resistance via regulating P-glycoprotein expression, the EMT process, and activation of phosphatidylinositol 3-kinase/protein kinase $\mathrm{B} /$ mammalian target of rapamycin (PI3K/AKT $/ \mathrm{m}-\mathrm{TOR}$ ) signaling pathway. MALAT1 might be a potential therapeutic target for the treatment of DDPresistant OSCC. ${ }^{41}$

The LSCC TU686 cell line excels in resisting the growth-curbing effects of 5-fluorouracil, paclitaxel, and vincristine compared with the TU177, AMC-HN-8, and LSC-1 cell lines (all $\mathrm{P}<0.05$ ). Moreover, there seems to be a great potential for overexpressed MALAT1 to enhance the chemoresistance of TU686 and LSC-1 cell lines $(\mathrm{P}<0.05)$. Silencing MALAT1 tends to undermine the proliferative and metastatic abilities of TU686 and LSC-1 cell lines $(\mathrm{P}<0.05)$. IncRNA MALAT1 is involved in triggering tolerance of LSCC against chemotherapeutics by enhancing and dampening tumor cell metastasis and apoptosis, respectively. ${ }^{42}$ MALAT1 is overexpressed in HNSCC cell lines compared to a nontumorigenic cell line. The number of colonies that form after radiation is significantly reduced in MALAT1 knockdown cells, and the half maximal inhibitory concentration of DDP in MALAT1 knockdown cells is also lower than in control cells. MALAT1 knockdown results in cell cycle arrest at G2/M phase, DNA damage and apoptotic cell death. MALAT1 knockdown enhances HNSCC cell sensitivity to radiation and DDP, partly through inducing G2/M cell cycle arrest resulting in DNA damage and apoptosis. ${ }^{42}$

Jiang et al evaluated the involvement of lncRNA MALAT1 in modifying LSCC cell line chemosensitivity. lncRNA MALAT1 renders LSCC less sensitive to chemotherapeutics by boosting tumor cell metastasis and depressing apoptosis of tumor cells. ${ }^{43}$ 


\section{The Role of MALATI in HNSCC} Treatment

Studies have shown that MALAT1 and related genes and proteins are targets of therapies that can inhibit HNSCC cell proliferation and metastasis and provide new ideas for tumor treatment.

MALAT1 expression is upregulated in HNSCC cell lines compared to non-tumorigenic cell lines. ${ }^{42}$ Authors first examined the expression of MALAT1 in two HNSCC cell lines ( $\mathrm{Ca} 9-22$ and FaDu) as compared to non-tumorigenic human skin keratinocyte cell line (HaCaT) using reverse transcription quantitative polymerase-chain reaction (qRT-PCR). They found that MALAT1 expression is significantly higher in $\mathrm{Ca} 9-22$ $(\mathrm{P}<0.05)$ and FaDu $(\mathrm{P}<0.0001)$ cells compared to HaCaT cells. The number of MALAT1 knockdown cell colonies formed after radiation is also significantly reduced. MALAT1 gene knockdown can enhance the sensitivity of HNSCC cells to radiotherapy and DDP. Interfering with MALAT1 expression in head and neck tumors can inhibit tumor growth and metastasis and increase tumor cell apoptosis and sensitivity to radiotherapy. $^{44}$

Inactivation of MALAT1 can inhibit gene expression regulators that modify the characteristics of laryngeal cancer and hypopharyngeal carcinoma, and blocking MALAT1 expression can inhibit the progression of both types of cancer. ${ }^{33}$ IncRNA MALAT1 can trigger LSCC resistance to chemotherapy drugs by promoting tumor cell metastasis and inhibiting apoptosis. ${ }^{43}$ lncRNA MALAT1 serves as a miR-429 sponge, regulating ZEB1 expression. MALAT1 inhibition leads to increased miR-429 and suppression of hypopharyngeal squamous cell carcinoma (HSCC) progression via ZEB1 reduction, providing a potential therapeutic target for HSCC. ${ }^{45}$ MALAT1 expression levels were determined using RT-qPCR, and cell counting kit-8, transwell, and flow cytometry assays were performed to investigate the biological functions of HSCC cells. The results indicated MALAT1 upregulation in HSCC. MALAT1 knockdown suppresses the malignant behavior of HSCC by targeting miR-194, and MALAT1 is expected to become a new therapeutic target for HSCC. ${ }^{46}$

In nasopharyngeal carcinoma (NPC), MALAT1 regulates cancer stem cell (CSC) activity and radioresistance by modulating the miR-1/Slug axis, indicating that MALAT1 may also be a therapeutic target for patients with NPC. ${ }^{47}$
MALAT1 is one of the most widely studied lncRNAs related to various human cancers. Yu et $\mathrm{al}^{48}$ discussed the role of MALAT1 in OSCC and the possible regulatory mechanisms involved. They showed that MALAT1 and miR-143-3p expression were negatively correlated and that MALAT1 expression was positively correlated with that of MAGEA9. An inverse correlation between MAGEA9 and miR-143-3p expression was also observed. These results show that MALAT1 is a promising new therapeutic target for OSCC treatment. Others have shown that MALAT1 is upregulated in OSCC cell lines. Inhibition of MALAT1 can prevent OSCC proliferation, whereas overexpressing MALAT1 promotes OSCC progression. The function of MALAT1 in OSCC development was further investigated with in vivo assays. Studies in established nude mice models revealed that downregulated MALAT1 greatly inhibited OSCC tumor growth; conversely, upregulated MALAT1 promoted OSCC development via the miR-125b/STAT3 axis. MALAT1 can regulate STAT3 expression in OSCC by absorbing miR-125b. ${ }^{49}$ MALAT1, SRSF1, and CCR7 have exceptionally high expression levels in samples from patients with OSCC with lymphatic metastasis compared to samples without lymph node metastasis as well as paracarcinoma tissues, exclusive of miRNA-320d levels. Interestingly, the target RNAs (MALAT-1, miRNA-320s) and SRSF1 protein can combine with each other, based on the statistically significant difference compared with negative control group $(\mathrm{P}<0.05)$. CCR7 expression was higher than the negative control group after silencing miRNA320d. MALAT-1 interacts with miRNA-320d through SRSF1 and is expected to be a useful target for OSCC prevention and treatment. ${ }^{50}$ Xiao et al $^{51}$ explored the biological functions and potential mechanism of MALAT1 in OSCC. Functional studies revealed that MALAT1 overexpression promotes OSCC cell proliferation and invasion. Further experiments revealed that miR-101 is a MALAT1 target, and an miR-101 inhibitor abolishes the effect of MALAT1 on OSCC cell proliferation and invasion. Enhancer of zeste 2 polycomb repressive complex 2 subunit (EZH2) acted as a downstream effector of MALAT1 in the OSCC cells.

MALAT-1 expression is enhanced in TSCCs, especially in those cases with lymph node metastasis. MALAT-1 knockdown in TSCC cells impairs migration and proliferation in vitro and leads to fewer metastases in vivo. DNA microarray analysis revealed up-regulated expression of several small proline-rich proteins (SPRRs) in TSCC cells following MALAT-1 
knockdown. Moreover, SPRR2A over-expression impairs distant TSCC cell metastasis in vivo. ${ }^{52}$ MALAT1 and p21 (RAC1)-activated kinase 1 (PAK1) are upregulated and miR-140-5p expression is downregulated in TSCC tissues and cells. MALAT1 knockdown induces miR-140-5p expression by direct interaction. Moreover, MALAT1 knockdown inhibits TSCC cell proliferation, migration, and invasion by upregulating miR140-5p expression. ${ }^{53}$ MALAT1 knockdown suppresses the growth and invasion of human TSCC cells and inhibits metastasis in vitro and in vivo. In addition, miR124-dependent jagged1 (JAG1) regulation is required for MALAT1-induced TSCC cell growth. MALAT1 may have a carcinogenic effect by increasing TSCC proliferation and metastasis, and is therefore a potential therapeutic target. $^{54}$

\section{Regulatory Factors and the Mechanism of MALATI in HNSCC}

Clarification of biological functions is the basis for identifying ideal tumor markers. Therefore, understanding how MALAT1 affects the growth of HNSCC cells at the mechanistic level will allow us to determine whether MALAT1 can be a useful diagnostic marker and therapeutic target for HNSCC. The occurrence and development of HNSCC is a complex process involving multiple factors, several stages, and the accumulation of mutations in various genes. The mechanisms underlying immortal proliferation of tumor cells are very complicated and include inactivation of tumor suppressor genes, overexpression of oncogenes, loss of cell cycle control, cell cycle failure, and tolerance to apoptosis. A number of studies have shown that a variety of regulatory factors regulate the expression of MALAT1 in HNSCC, including transforming growth factor (TGF)- $\beta$ / miRNA-124/ERK/MAPK, SRSF1, MEK, and BI-847325. MALAT1 can promote oncogene expression and cell cycle progress and inhibit tumor cell apoptosis to promote tumor growth. HNSCC cells rapidly proliferate following protooncogene activation. MALAT1 can target multiple oncogenes or proteins to promote their expression and exert growth-promoting effects, including miR-429/ZEB1, miR124/Capn4, PI3K/AKT/m-TOR, and Wnt/ $\beta$-catenin.

\section{Regulatory Factors of MALATI in HNSCC}

$\mathrm{Du}$ et $\mathrm{al}^{55}$ showed that constant TGF- $\beta$ stimulation represses miR-124 expression, whereas miR-124 overexpression antagonizes TGF- $\beta$-promoted NPC cell growth and migration. miR-124 overexpression decreases p-SMAD2/3, SMAD4, and p-ERK levels, indicating that ectopic miR-124 overexpression inhibits SMAD and nonSMAD pathways. Pro-oncogenic lncRNA MALAT1 is targeted by miR-124 that regulates ERK/MAPK by targeting MALAT1, independently of the SMAD signaling pathway. TGF- $\beta$ can regulate the ERK/MAPK pathway without depending on SMAD by inhibiting miRNA-124. This in turn increases MALAT1 expression in NPC.

In OSCC, SRSF1 can mediate the interaction between MALAT1 and miRNA-320d. Silencing miRNA-320d significantly increases CCR7 expression. Together, these data reveal the molecular-level effect of MALAT1 on the CCR7 chemokine receptor, indicating that MALAT1 may further affect lymph node metastasis of OSCC. ${ }^{50}$ The MALAT1 regulatory factors in different types of HNSCC are shown in Table 1.

\section{The Mechanism of MALATI in HNSCC}

Kangboonruang et $\mathrm{al}^{42}$ demonstrated that knocking down MALAT1 gene expression can induce DNA damage and apoptosis by inducing $\mathrm{G} 2 / \mathrm{M}$ cell cycle arrest and enhancing the sensitivity of HNSCC cells to radiotherapy and chemotherapy.

MALAT1 and ZEB1 expression in HSCC are upregulated, while miR-429 expression is downregulated. Conversely, reduced MALAT1 and ZEB1 and upregulated miR-429 inactivate the PI3K/Akt/mTOR signaling pathway; suppress in vitro viability, colony formation, migration, and invasion; decrease cell growth in vivo; and promote the apoptosis of $\mathrm{FaDu}$ cells. Downregulating miR-429 reverses the role of MALAT1 inhibition in FaDu cell growth. IncRNA MALAT1 serves as a sponge of miR-429, thus regulating ZEB1 expression. MALAT1 inhibition can inhibit HSCC progression by increasing miR-429 and decreasing ZEB1. ${ }^{45}$ MALAT1 knockdown suppresses HSCC cell proliferation, migration, and invasion and promotes apoptosis compared with the control group. Additionally, miR-194 was identified as a target of MALAT1 expressed at low levels in HSCC tissues

Table I MALATI Regulatory Factors in HNSCC

\begin{tabular}{|l|l|l|}
\hline Tumor Type & Target Molecule & Reference \\
\hline NPC & TGF- $\beta /$ miRNA-I24/ERK/MAPK & {$[55]$} \\
OSCC & SRSFI & {$[50]$} \\
\hline
\end{tabular}

Abbreviations: NPC, nasopharyngeal carcinoma; OSCC, oral squamous cell carcinoma. 
compared with adjacent non-tumor tissues. An miR-194 agomir inhibits malignant cell behaviors including proliferation, migration and invasion, whereas an miR-194 antagomir promotes malignant behaviors compared with the corresponding control groups. MALAT1 knockout can also inhibit HSCC progression by targeting miR-194. ${ }^{46}$ Inhibition of miR-194 partially reverses MALAT1 knockdown-induced inhibitory effects in HSCC cells.

Shi et $\mathrm{al}^{56}$ showed that MALAT1 absorbs miR-124 through a sponge effect; inhibits Capn4; and promotes the proliferation, invasion, and EMT of NPC cells. This revealed a new MALAT1/miR-124/Capn4 regulatory axis involved in NPC pathogenesis. MALAT1 can also be used as a competitive endogenous RNA (ceRNA) in NPC to increase Slug mRNA and protein levels. The combination of MALAT1 and miRNA-1 reduces miRNA-1 and Slug mRNA levels, while MALAT1 can eliminate miRNA-1 induced Slug gene silencing. ${ }^{47}$

With regard to OSCC, two kinds of human DDPresistant cell lines (CAL-27R and SCC-9R) were developed from DDP-naïve cell lines (CAL-27 and SCC-9, respectively) as in vitro cell models. Cell transfection was performed to overexpress or knockdown MALAT1, and mouse xenograft models were established. The following measurements were performed: cell proliferation, colony formation, wound healing, transwell, and terminal deoxynucleotidyl transferase dUTP nick end labeling assays, as well as Western blotting and immunofluorescence staining. MALAT1 can promote the EMT and enhance resistance to DDP through the $\mathrm{PI} 3 \mathrm{~K} / \mathrm{AKT} /$ m-TOR signaling pathway. ${ }^{41}$ Yu et $\mathrm{al}^{48}$ discussed the role of MALAT1 in OSCC and possible regulatory mechanisms and showed that MALAT1 acts as a ceRNA to promote OSCC cell proliferation and migration through the miR-143-3p/MAGEA9 axis. A bioinformatics search revealed that miR-125b is a direct target of MALAT1, suggesting a negative correlation between MALAT1 and miR-125b. STAT3 was also predicted as a binding target of miR-125b in OSCC. Overexpression of MALAT1 was able to suppress the tumor inhibitory effect of miR-125b mimics by upregulating STAT3. MALAT1 can regulate STAT3 expression by absorbing miR-125b to promote OSCC occurrence and development. ${ }^{49}$ Xiao et al $^{51}$ performed qPCR analyses and found obviously increased MALAT1 expression and decreased miR-101 in OSCC tissues and cell lines. This suggests that upregulation of

Table 2 MALATI Target Molecules and Consequences of Their Targeting in HNSCC

\begin{tabular}{|c|c|c|c|}
\hline Tumor Type & Target Molecule & Effect & Reference \\
\hline HNSCC & $\mathrm{G}_{2} / \mathrm{M}$ & Sensitivity & [42] \\
\hline \multirow[t]{2}{*}{ HSCC } & miR-429/ZEBI & Differentiation, proliferation, invasion, metastasis & {$[45]$} \\
\hline & miR-194 & Differentiation, proliferation, invasion, metastasis & [46] \\
\hline \multirow[t]{2}{*}{ NPC } & miR-I24/Capn4 & Proliferation, invasion, EMT & {$[56]$} \\
\hline & miRNA-I/Slug mRNA & Differentiation, proliferation, invasion, metastasis & [47] \\
\hline \multirow[t]{5}{*}{ OSCC } & $\mathrm{PI} 3 \mathrm{~K} / \mathrm{AKT} / \mathrm{m}-\mathrm{TOR}$ & EMT, sensitivity & {$[4 I]$} \\
\hline & miR-143-3p/MAGEA9 & Proliferation, invasion, metastasis & [48] \\
\hline & miR-125b/STAT3 & Differentiation, proliferation, invasion, metastasis & {$[49]$} \\
\hline & CCR7 & Metastasis & [50] \\
\hline & miR-I0I/EZH2 & Proliferation, invasion & [51] \\
\hline \multirow[t]{5}{*}{ TSCC } & Wnt/R-catenin & EMT, apoptosis & [57] \\
\hline & PI3K/Akt, MMP-9 & Proliferation, invasion, metastasis & [58] \\
\hline & SPRR & Metastasis & {$[52]$} \\
\hline & miR-140-5p-PAKI & Proliferation, invasion, metastasis & [53] \\
\hline & miR-|24/JAG I & Proliferation, metastasis & {$[54]$} \\
\hline
\end{tabular}

Abbreviations: HNSCC, head and neck squamous cell carcinoma; HSCC, hypopharyngeal squamous cell carcinoma; NPC, nasopharyngeal carcinoma; OSCC, oral squamous cell carcinoma; TSCC, tongue squamous cell carcinoma. 
MALAT1 can promote OSCC proliferation and invasion by targeting the miR-101/EZH2 axis.

lncRNA MALAT1 is upregulated in TSCC tissues and correlates with cervical lymph node metastasis in patients with TSCC. Moreover, MALAT1 induces cell migration, invasion, and EMT and inhibits apoptosis by modulating Wnt $/ \beta$-catenin signaling. The effect of exogenous MALAT1 is also inhibited by the Wnt/ $\beta$-catenin signaling pathway. In TSCC, MALAT1 promotes the EMT and inhibits apoptosis by regulating the $\mathrm{Wnt} / \beta$-catenin signaling pathway. ${ }^{57}$ Yuan et al ${ }^{58}$ studied the function of MALAT1 in TSCC and found that MALAT1 expression was remarkably higher in tumor tissues compared to adjacent healthy tissues. Serum MALAT1 was significantly higher in patients with TSCC versus healthy controls. MALAT1 knockdown markedly inhibits TSCC cell proliferation, migration, and invasion. MALAT1 knockdown also reduces Akt phosphorylation levels and matrix metalloproteinase-9 expression. High MALAT1 expression is related to TSCC growth and metastasis, but MALAT1

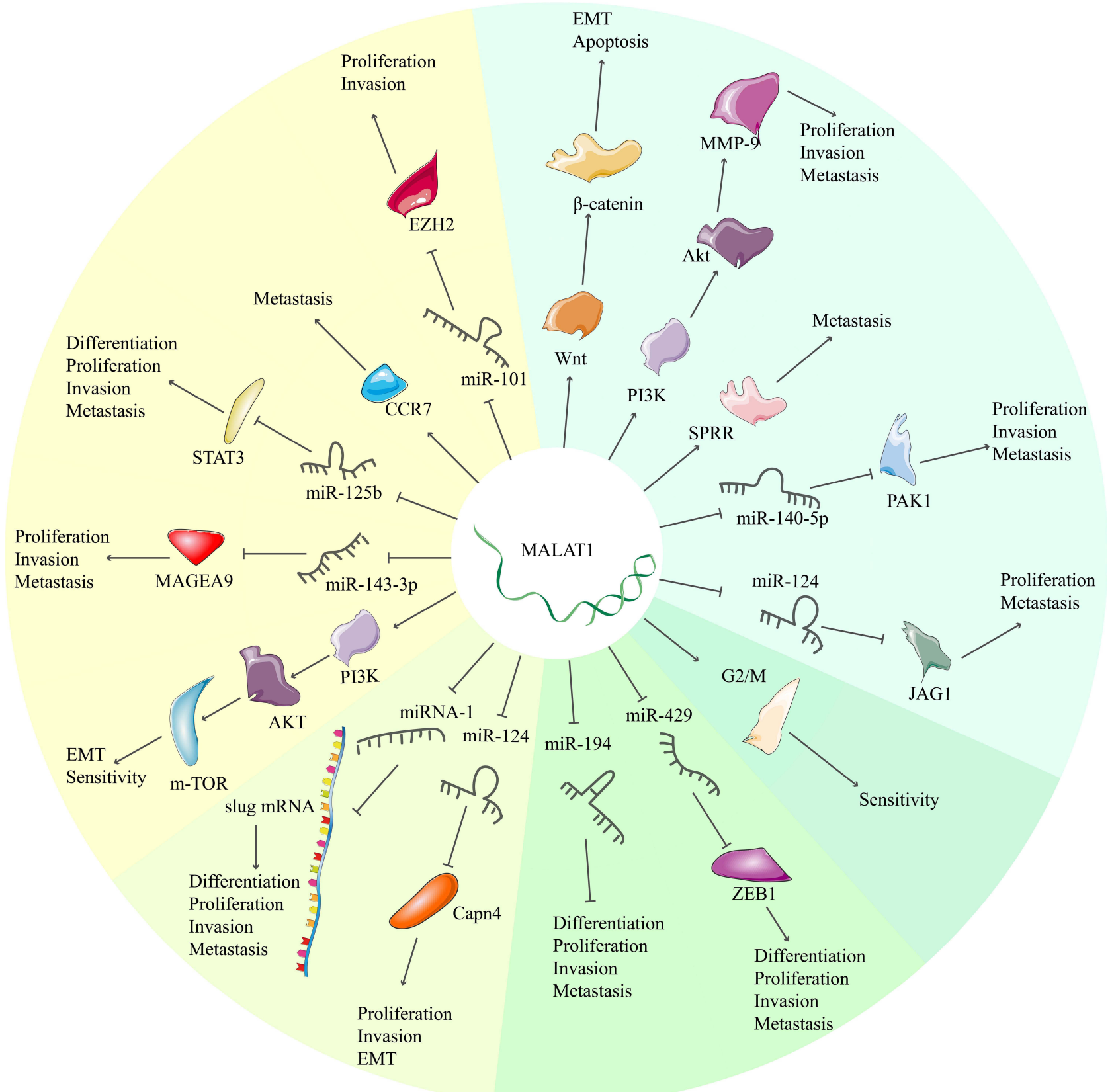

Figure I The mechanisms of MALATI in HNSCC. 
knockout can upregulate some SPRR proteins, thereby affecting the distant metastasis of TSCC cells. ${ }^{52}$ The expression of MALAT1 and p21 (RAC1)-activated kinase 1 (PAK1) are upregulated and miR-140-5p expression is downregulated in TSCC tissues and cells. MALAT1 can promote TSCC progression through the miR-140-5pPAK1 regulatory axis. ${ }^{53}$ Zhang et al $^{54}$ showed that MALAT1 interacts with miR-124 to regulate TSCC growth by targeting JAG1, and that MALAT1 exerts carcinogenic effects by increasing TSCC proliferation and metastasis. MALAT1 target molecules vary in different types of HNSCC. The target molecules and effects of MALAT1 in common types of HNSCC are shown in Table 2. The mechanisms of MALAT1 in HNSCC are shown in Figure 1.

\section{Conclusion}

Abnormal MALAT1 expression plays an important role in HNSCC occurrence and development. In most types of HNSCC, higher MALAT1 expression is associated with poorer prognosis. With increased MALAT1-focused research, additional roles and mechanisms will gradually be elucidated, and these will have implications for HNSCC diagnosis, treatment, and prognosis. With continuous advancement of biological science and technology including more accurate sequencing methods, future studies exploring the precise and detailed mechanism of MALAT1 action and function will aid in the early diagnosis of HNSCC and identify new treatment targets and biomarkers. Together, these advances are likely to lead to the establishment of personalized precision medical treatment and improved prognostic evaluation for patients with HNSCC.

\section{Acknowledgments}

This work was supported by grants from the Natural Science Foundation of Zhejiang Provincial (Grant Nos. LY19H160014, LY20H130001, LQ21H130001), Medical and Health Research Project of Zhejiang Province (Grant Nos. 2019ZD018, 2021KY307), Ningbo Health Branding Subject Fund (Grant No. PPXK2018-02), Ningbo Natural Science Foundation (Grant Nos. 2018A610361, 2019A610319, 202003N4239), and Ningbo "Technology Innovation 2025" Major Special Project (Grant Nos. 2018B10015, 2020Z097).

\section{Disclosure}

The authors report no conflicts of interest in this work.

\section{References}

1. Johnson DE, Burtness B, Leemans CR, Lui VWY, Bauman JE, Grandis JR. Head and neck squamous cell carcinoma. Nat Rev Dis Primers. 2020;6(1):92.

2. Siegel RL, Miller KD, Jemal A. Cancer Statistics, 2017. CA Cancer J Clin. 2017;67(1):7-30. doi:10.3322/caac.21387

3. Liu C, Yu Z, Huang S, et al. Combined identification of three miRNAs in serum as effective diagnostic biomarkers for HNSCC. EBioMedicine. 2019;50:135-143. doi:10.1016/j.ebiom.2019.11.016

4. Cramer JD, Burtness B, Le QT, Ferris RL. The changing therapeutic landscape of head and neck cancer. Nat Rev Clin Oncol. 2019;16 (11):669-683. doi:10.1038/s41571-019-0227-z

5. Denaro N, Russi EG, Merlano MC. Pros and cons of the new edition of TNM classification of head and neck squamous cell carcinoma. Oncology. 2018;95(4):202-210. doi:10.1159/000490415

6. Kolenda T, Guglas K, Kopczynska M, et al. Oncogenic role of ZFAS1 lncRNA in head and neck squamous cell carcinomas. Cells. 2019;8(4):366. doi:10.3390/cells8040366

7. Leemans CR, Snijders PJF, Brakenhoff RH. The molecular landscape of head and neck cancer. Nat Rev Cancer. 2018;18(5):269-282. doi:10.1038/nrc.2018.11

8. von Witzleben A, Wang C, Laban S, Savelyeva N, Ottensmeier CH. HNSCC: tumour antigens and their targeting by immunotherapy. Cells. 2020;9(9):2103. doi:10.3390/cells9092103

9. Ali T, Grote P. Beyond the RNA-dependent function of LncRNA genes. eLife. 2020;9:e60583. doi:10.7554/eLife.60583

10. Chen Q, Zhu C, Jin Y. The oncogenic and tumor suppressive functions of the long noncoding RNA MALAT1: an emerging controversy. Front Genet. 2020;11:93. doi:10.3389/fgene.2020.00093

11. Li W, Chen Y, Nie X. Regulatory mechanisms of lncRNAs and their target gene signaling pathways in laryngeal squamous cell carcinoma. Front Pharmacol. 2020;11:1140. doi:10.3389/fphar.2020.01140

12. Robinson EK, Covarrubias S, Carpenter S. The how and why of lncRNA function: an innate immune perspective. Biochim Biophys Acta Gene Regul Mech. 2020;1863(4):194419.

13. Pacholewska A, Sung MH. IncRNA expression predicts mRNA abundance. Epigenomics. 2019;11(10):1121-1128. doi:10.2217/epi2019-0003

14. Chi Y, Wang D, Wang J, Yu W, Yang J. Long non-coding RNA in the pathogenesis of cancers. Cells. 2019;8(9):1015. doi:10.3390/ cells 8091015

15. Wang Y, Wang S, Ren Y, Zhou X. The role of lncRNA crosstalk in leading cancer metastasis of head and neck squamous cell carcinoma. Front Oncol. 2020;10:561833. doi:10.3389/fonc.2020.561833

16. Akbari Dilmaghani N, Khoshsirat S, Shanaki-Bavarsad M, Pourbagheri-Sigaroodi A, Bashash D. The contributory role of long non-coding RNAs (lncRNAs) in head and neck cancers: possible biomarkers and therapeutic targets? Eur $J$ Pharmacol. 2021;900:174053. doi:10.1016/j.ejphar.2021.174053

17. Meng X, Wang ZF, Lou QY, et al. Long non-coding RNAs in head and neck squamous cell carcinoma: diagnostic biomarkers, targeted therapies, and prognostic roles. Eur J Pharmacol. 2021;902:174114. doi:10.1016/j.ejphar.2021.174114

18. Huang F, Xin C, Lei K, Bai H, Li J, Chen Q. Noncoding RNAs in oral premalignant disorders and oral squamous cell carcinoma. Cell Oncol (Dordr). 2020;43(5):763-777. doi:10.1007/s13402-02000521-9

19. Zhao J, Liu D, Yang H, Yu S, He H. Long noncoding RNAs in head and neck squamous cell carcinoma: biological functions and mechanisms. Mol Biol Rep. 2020;47(10):8075-8090. doi:10.1007/ s11033-020-05777-w

20. Arun G, Aggarwal D, Spector DL. MALAT1 long non-coding RNA: functional implications. Noncoding RNA. 2020;6(2):22. doi:10.3390/ ncrna6020022 
21. Sun Y, Ma L. New insights into long non-coding RNA MALAT1 in cancer and metastasis. Cancers (Basel). 2019;11(2):216. doi:10.3390/ cancers 11020216

22. Yao Y, Chen X, Lu S, et al. Circulating long noncoding RNAs as biomarkers for predicting head and neck squamous cell carcinoma. Cell Physiol Biochem. 2018;50(4):1429-1440. doi:10.1159/ 000494605

23. Malakar P, Shilo A, Mogilevsky A, et al. Long noncoding RNA MALAT1 promotes hepatocellular carcinoma development by SRSF1 upregulation and mTOR activation. Cancer Res. 2017;77 (5):1155-1167. doi:10.1158/0008-5472.CAN-16-1508

24. Wang ML, Liu JX. MALAT1 rs619586 polymorphism functions as a prognostic biomarker in the management of differentiated thyroid carcinoma. J Cell Physiol. 2020;235(2):1700-1710. doi:10.1002/ jcp. 29089

25. Arun G, Spector DL. MALAT1 long non-coding RNA and breast $\begin{array}{lll}\text { cancer. } & \text { RNA Biol. 2019;16(6):860-863. doi:10.1080/ }\end{array}$ 15476286.2019.1592072

26. Voce DJ, Bernal GM, Wu L, et al. Temozolomide treatment induces IncRNA MALAT1 in an NF-kappaB and p53 codependent manner in glioblastoma. Cancer Res. 2019;79(10):2536-2548. doi:10.1158/ 0008-5472.CAN-18-2170

27. Liu C, Li H, Jia J, Ruan X, Liu Y, Zhang X. High metastasis-associated lung adenocarcinoma transcript 1 (MALAT1) expression promotes proliferation, migration, and invasion of non-small cell lung cancer via ERK/Mitogen-Activated Protein Kinase (MAPK) signaling pathway. Med Sci Monit. 2019;25:5143-5149. doi:10.12659/MSM.913308

28. Qiao Y, Peng C, Li J, Wu D, Wang X. LncRNA MALAT1 is neuroprotective in a rat model of spinal cord ischemia-reperfusion injury through miR-204 regulation. Curr Neurovasc Res. 2018;15 (3):211-219. doi:10.2174/1567202615666180712153150

29. Chen R, Liu Y, Zhuang H, et al. Quantitative proteomics reveals that long non-coding RNA MALAT1 interacts with $\mathrm{DBC1}$ to regulate $\mathrm{p} 53$ acetylation. Nucleic Acids Res. 2017;45(17):9947-9959. doi:10.1093/ nar/gkx600

30. Lin Q, Guan W, Ren W, Zhang L, Zhang J, Xu G. MALAT1 affects ovarian cancer cell behavior and patient survival. Oncol Rep. 2018;39 (6):2644-2652. doi:10.3892/or.2018.6384

31. Wang WW, Zhou XL, Song YJ, Yu CH, Zhu WG, Tong YS. Combination of long noncoding RNA MALAT1 and carcinoembryonic antigen for the diagnosis of malignant pleural effusion caused by lung cancer. Onco Targets Ther. 2018;11:2333-2344. doi:10.2147/ OTT.S157551

32. Garo Kyurkchiyan S, Miroslavov Popov T, Stancheva G, et al. Novel insights into laryngeal squamous cell carcinoma from association study of aberrantly expressed miRNAs, lncRNAs and clinical features in Bulgarian patients. J BUON. 2020;25 (1):357-366.

33. Xu E, Liang X, Ji Z, Zhao S, Li L, Lang J. Blocking long noncoding RNA MALAT1 restrained the development of laryngeal and hypopharyngeal carcinoma. Eur Arch Otorhinolaryngol. 2020;277 (2):611-621. doi:10.1007/s00405-019-05732-X

34. Wang S, Wang T, Liu D, Kong H. LncRNA MALAT1 aggravates the progression of non-small cell lung cancer by stimulating the expression of COMMD8 via targeting miR-613. Cancer Manag Res. 2020;12:10735-10747. doi:10.2147/CMAR.S263538

35. Zhao L, Lou G, Li A, Liu Y. IncRNA MALAT1 modulates cancer stem cell properties of liver cancer cells by regulating YAP1 expression via miR375 sponging. Mol Med Rep. 2020;22(2):1449-1457. doi:10.3892/mmr.2020.11196

36. Wang K, Zhao Y, Wang YM. LncRNA MALAT1 promotes survival of epithelial ovarian cancer cells by downregulating miR-145-5p. Cancer Manag Res. 2020;12:11359-11369. doi:10.2147/CMAR. S267355
37. He B, Zeng J, Chao W, et al. Serum long non-coding RNAs MALAT1, AFAP1-AS1 and AL359062 as diagnostic and prognostic biomarkers for nasopharyngeal carcinoma. Oncotarget. 2017;8 (25):41166-41177. doi:10.18632/oncotarget.17083

38. Han X, Xu Z, Tian G, et al. Suppression of the long non-coding RNA MALAT-1 impairs the growth and migration of human tongue squamous cell carcinoma SCC4 cells. Arch Med Sci. 2019;15(4):992-1000. doi:10.5114/aoms.2018.73343

39. Hu Y, Zheng L, Zhang J, Shen Y, Zhang X, Lin L. LncRNAMALAT1 is a promising biomarker for prognostic evaluation of tongue squamous cell carcinoma. Eur Arch Otorhinolaryngol. 2020;277(11):3155-3160. doi:10.1007/s00405-020-06023-6

40. Vishwakarma S, Pandey R, Singh R, Gothalwal R, Kumar A. Expression of H19 long non-coding RNA is down-regulated in oral squamous cell carcinoma. J Biosci. 2020;45:145. doi:10.1007/ s12038-020-00118-2

41. Wang R, Lu X, Yu R. IncRNA MALAT1 promotes EMT process and cisplatin resistance of oral squamous cell carcinoma via PI3K/AKT/ m-TOR signal pathway. Onco Targets Ther. 2020;13:4049-4061. doi:10.2147/OTT.S251518

42. Kangboonruang $K$, Wongtrakoongate $P$, Lertsuwan $K$, et al. MALAT1 decreases the sensitivity of head and neck squamous cell carcinoma cells to radiation and cisplatin. Anticancer Res. 2020;40 (5):2645-2655. doi:10.21873/anticanres. 14235

43. Jiang Q, Liu S, Hou L, Guan Y, Yang S, Luo Z. The implication of LncRNA MALAT1 in promoting chemo-resistance of laryngeal squamous cell carcinoma cells. J Clin Lab Anal. 2020;34(4):e23116. doi:10.1002/jcla.23116

44. Li X, Cao Y, Gong X, Li H. Long noncoding RNAs in head and neck cancer. Oncotarget. 2017;8(6):10726-10740. doi:10.18632/ oncotarget. 12960

45. Liu X, Zhao W, Wang X. Inhibition of long non-coding RNA MALAT1 elevates microRNA-429 to suppress the progression of hypopharyngeal squamous cell carcinoma by reducing ZEB1. Life Sci. 2020;262:118480. doi:10.1016/j.1fs.2020.118480

46. Wang H, Wang F, Ouyang W, Jiang X, Li W. MALAT1 knockdown inhibits hypopharyngeal squamous cell carcinoma malignancy by targeting microRNA-194. Oncol Lett. 2020;20(1):173-182. doi:10.3892/ol.2020.11551

47. Jin C, Yan B, Lu Q, Lin Y, Ma L. The role of MALAT1/miR-1/slug axis on radioresistance in nasopharyngeal carcinoma. Tumour Biol. 2016;37(3):4025-4033. doi:10.1007/s13277-015-4227-z

48. Yu L, Shao X, Huo L, Zhang T. Long non-coding RNA (lncRNA) metastasis-associated lung adenocarcinoma transcript 1 (MALAT1) promotes cell proliferation and migration by regulating miR-143-3p and MAGE family member A9 (MAGEA9) in oral squamous cell carcinoma. Med Sci Monit. 2020;26:e924187. doi:10.12659/ MSM.924187

49. Chang SM, Hu WW. Long non-coding RNA MALAT1 promotes oral squamous cell carcinoma development via microRNA-125b/ STAT3 axis. J Cell Physiol. 2018;233(4):3384-3396. doi:10.1002/ jcp. 26185

50. Xu Z, Han X, Tang Z, Tian G, Gao J, Xu X. Interaction between MALAT-1, CCR7 and correlated genes in oral squamous cell carcinoma. Int J Clin Exp Pathol. 2017;10(11):10730-10739.

51. Xiao L, Wang W, Zhao J, Xu H, Li S, Yang X. lncRNA MALAT1 promotes cell proliferation and invasion by regulating the miR-101/ EZH2 axis in oral squamous cell carcinoma. Oncol Lett. 2020;20 (5):164. doi:10.3892/ol.2020.12024

52. Fang Z, Zhang S, Wang Y, et al. Long non-coding RNA MALAT-1 modulates metastatic potential of tongue squamous cell carcinomas partially through the regulation of small proline rich proteins. $B M C$ Cancer. 2016;16:706. doi:10.1186/s12885-016-2735-x 
53. Zhu M, Zhang C, Chen D, Chen S, Zheng H. IncRNA MALAT1 potentiates the progression of tongue squamous cell carcinoma through regulating miR-140-5p-PAK1 pathway. Onco Targets Ther. 2019;12:1365-1377. doi:10.2147/OTT.S192069

54. Zhang TH, Liang LZ, Liu XL, et al. Long non-coding RNA MALAT1 interacts with miR-124 and modulates tongue cancer growth by targeting JAG1. Oncol Rep. 2017;37(4):2087-2094. doi:10.3892/or.2017.5445

55. Du M, Chen W, Zhang W, et al. TGF-? regulates the ERK/MAPK pathway independent of the SMAD pathway by repressing miRNA-124 to increase MALAT1 expression in nasopharyngeal carcinoma. Biomed Pharmacother. 2018;99:688-696. doi:10.1016/j. biopha.2018.01.120

56. Shi B, Wang Y, Yin F. MALAT1/miR-124/Capn4 axis regulates proliferation, invasion and EMT in nasopharyngeal carcinoma cells. Cancer Biol Ther. 2017;18(10):792-800. doi:10.1080/ 15384047.2017.1373214
57. Liang J, Liang L, Ouyang K, Li Z, Yi X. MALAT1 induces tongue cancer cells' EMT and inhibits apoptosis through Wnt/beta-catenin signaling pathway. J Oral Pathol Med. 2017;46(2):98-105. doi:10.1111/jop.12466

58. Yuan J, Xu XJ, Lin Y, et al. LncRNA MALAT1 expression inhibition suppresses tongue squamous cell carcinoma proliferation, migration and invasion by inactivating PI3K/Akt pathway and downregulating MMP-9 expression. Eur Rev Med Pharmacol Sci. 2019;23 (1):198-206. doi:10.26355/eurrev_201901_16765

\section{Publish your work in this journal}

OncoTargets and Therapy is an international, peer-reviewed, open access journal focusing on the pathological basis of all cancers, potential targets for therapy and treatment protocols employed to improve the management of cancer patients. The journal also focuses on the impact of management programs and new therapeutic agents and protocols on patient perspectives such as quality of life, adherence and satisfaction. The manuscript management system is completely online and includes a very quick and fair peer-review system, which is all easy to use. Visit http://www.dovepress.com/ testimonials.php to read real quotes from published authors. 